\title{
Orbital Apex Syndrome and Carotid Artery Injury Associated with Transorbital Stab Injury
}

\author{
Dimitri Laurent, MD*, Brian Corlis, MD and Gregory JA Murad, MD \\ Lillian Wells Department of Neurosurgery, University of Florida, Florida, USA
}

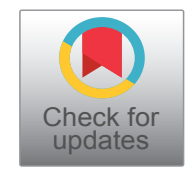

*Corresponding author: Dimitri Laurent, MD, Lillian Wells Department of Neurosurgery, University of Florida, McKnight Brain Institute, Room L2-100, 1149 South Newell Drive, Gainesville, Florida, 32611, USA, Tel: (352)-273-9000, Fax: (352)392-8413

\begin{abstract}
Transorbital penetrating injuries are particularly rare in the adult population. We present a case of TOPI sustained in an assault using a ballpoint pen-with concomitant transmaxillary injury due to a pencil stab wound-and a review of the literature.
\end{abstract}

\section{Introduction}

Transorbital penetrating injuries (TOPI) are particularly rare in the adult population, accounting for $0.096 \%$ of all head injuries [1-4]. In the setting of TOPI, there should be a high index of suspicion for damage to the intracranial vascular and neural structures. The conical orbital shape allows for penetrating objects to enter the intracranial space without fracture through three posterior apertures: The superior orbital fissure, optic canal, and the inferior orbital fissure [1-8]. We present a case of TOPI sustained in an assault using a ballpoint pen. We highlight the relevant anatomy, and associated neurological deficits, followed by a review of the relevant literature.

\section{Case Presentation}

A 53-year-old male inmate sustained a transorbital penetrating injury with a ballpoint pen, and a transmaxillary penetrating wound injury with a pencil. He was transferred to our university hospital fully alert, with stable vital signs, and GCS score of 13 (E3, V4, M6). On presentation, only the pen cap was visible, extending outward from his right medial canthal fold (Figure 1). Physical exam demonstrated right sided ophthalmople- gia, with absent pupillary responses to both direct and consensual light, and loss of light perception in the right eye. Left sided extraocular movements remained intact, and the left pupil was reactive to direct but not consensual light. His neurological exam was otherwise, normal. A computed tomography angiography (CTA) scan of the head demonstrated the pen entering the right medial orbit, extending intracranially through the superior orbital fissure along the lateral wall of the right cavernous sinus, and terminating supratentorially in the right temporal lobe (Figure 2A and Figure 2B). There was no evidence of hemorrhage along the track, and the right internal carotid artery was separated from the pen by two millimeters. A second tubular foreign body was seen beginning at the anterior aspect of the left masseter muscle, entering the lateral wall of the left maxillary sinus, and then passing through the medial wall of the sinus, inferior turbinate, nasal septum, and right ostiomeatal complex before terminating in the inferolateral right orbital wall (Figure 2C).

The patient was taken to the operating theater on the same day for surgical removal of the intracranial foreign body under direct microscopic visualization. The patient's neck was sterilized and draped to allow for quick access to the cervical carotid artery in the event of uncontrolled intraoperative hemorrhage. The intracranial compartment was accessed via a right pterional approach. After opening the Sylvian fissure, a brain ribbon was placed to retract the superomedial portion of the temporal lobe, and writing was identified on the pen. There was an adventitial injury to the carotid ar-

Citation: Laurent D, Corlis B, Murad GJA (2020) Orbital Apex Syndrome and Carotid Artery Injury Associated with Transorbital Stab Injury. Neurosurg Cases Rev 3:049. doi.org/10.23937/26434474/1710049

Accepted: December 07, 2020; Published: December 09, 2020

Copyright: (C) 2020 Laurent D, et al. This is an open-access article distributed under the terms of the Creative Commons Attribution License, which permits unrestricted use, distribution, and reproduction in any medium, provided the original author and source are credited. 

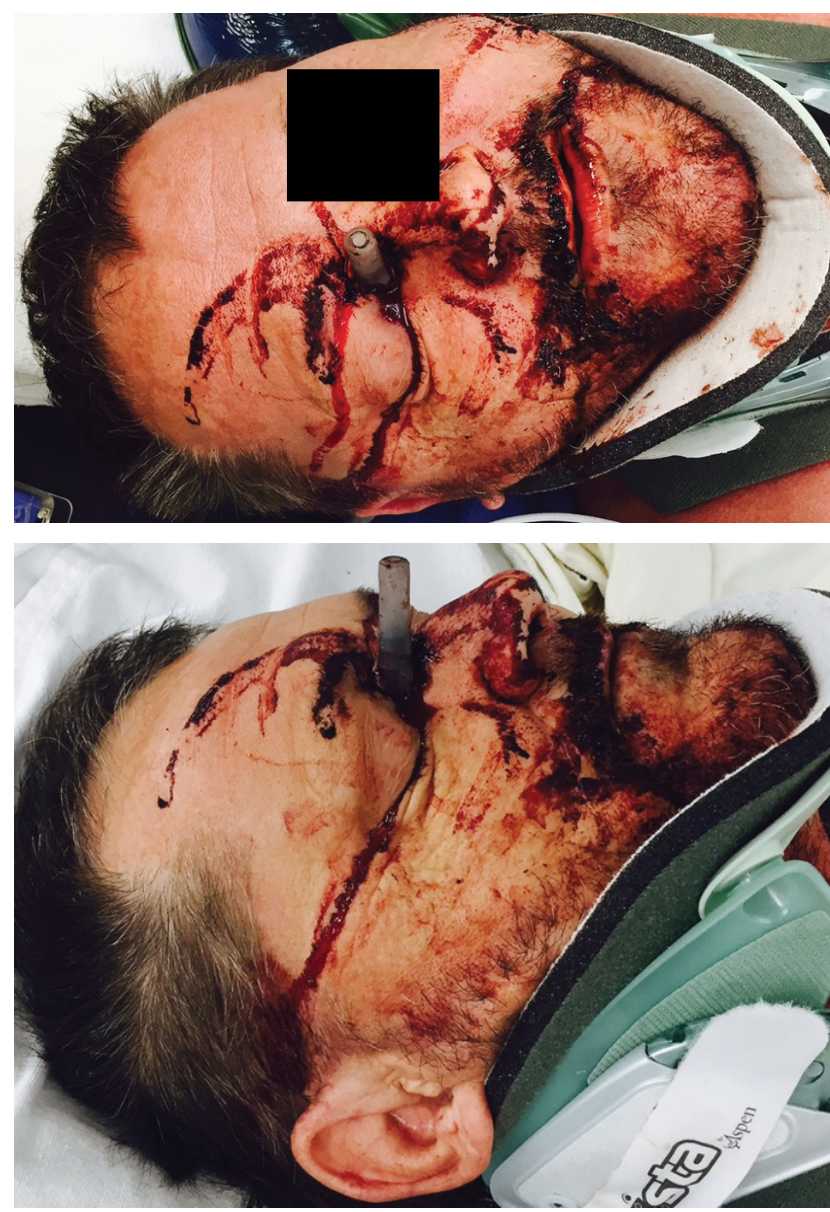

Figure 1: $(A, B)$ Gross picture of transorbital penetrating injury with the pen in situ.

tery that was not previously recognized on CTA. The trajectory of the pen into the brain through the superior orbital fissure was clearly visualized, and we were able to back the pen out about two centimeters. With the tip safely separated from the adjacent neurovascular structures, a second surgeon carefully removed the pen through the orbit from under the drapes (Figure 3). The removal was directly visualized under the operative microscope. There was some venous bleeding, controlled using Surgifoam ${ }^{\circledR}$ (Ethicon US, LLC; Somerville, NJ, USA), Gelfoam ${ }^{\circledast}$ (Pfizer; New York, NY, USA), and Surgicel ${ }^{\circledR}$ (Ethicon US, LLC; Somerville, NJ, USA). After copious irrigation, a large perforation in the medial dura was closed primarily without cerebrospinal fluid leak. The pencil was removed by the oral and maxillofacial surgery team after closure of the cranial wound.

Post-operatively, he was admitted to the neurosurgical intensive care unit and started on intravenous ampicillin/sulbactam. He had persistent right sided ophthalmoplegia with ptosis, and complete loss of light perception in the right eye. Left sided extraocular movements, pupillary function, and visual acuity remained intact. There was reduced sensation in the ophthalmic distribution of the right trigeminal nerve that was not appreciated preoperatively. Postoperative CTA demonstrated complete removal of both foreign bodies, with no evi-

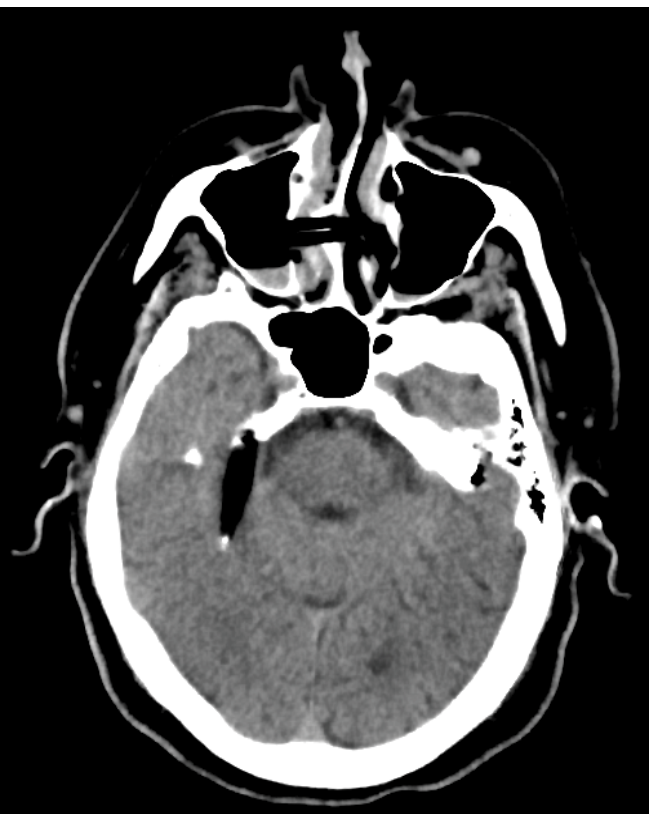

A.

B.
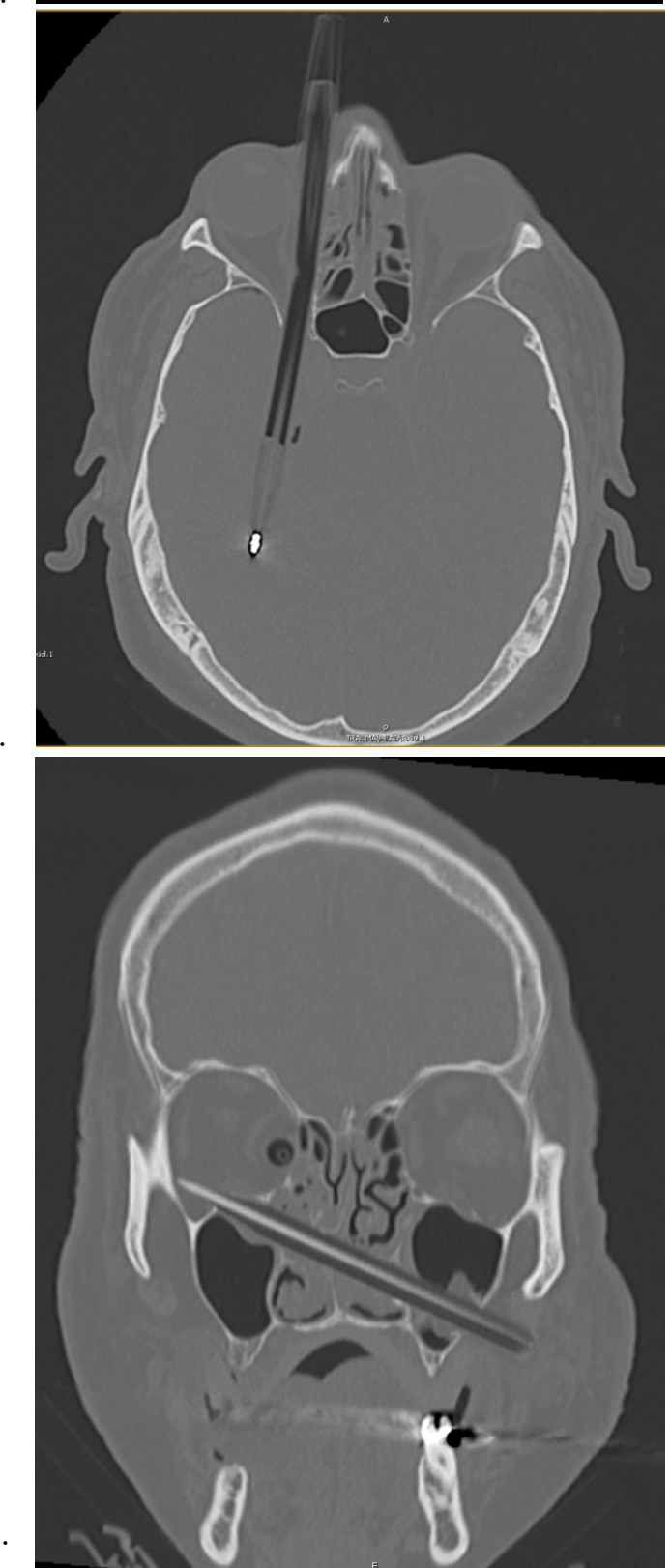

Figure 2: $(A, B, C)$ Brain and Bone window axial and coronal $\mathrm{CT}$ scans demonstrating ball point pen and pencil trajectory. 


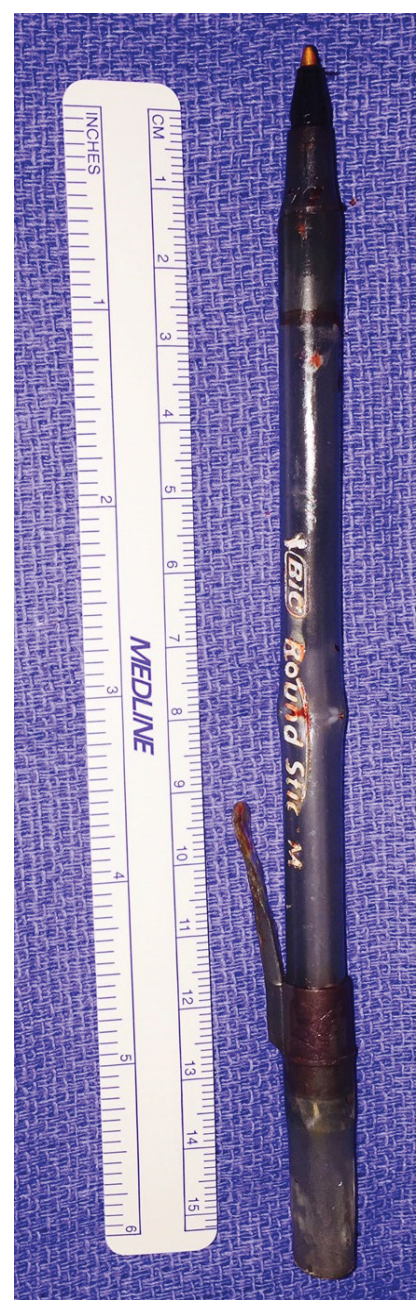

Figure 3: Pen following removal from the orbit.

dence of vascular injury (Figure 4A and Figure 4B). There was some edema of the right cerebral hemisphere, with about four millimeters of midline shift. He remained in ICU for about 24 hours before being transferred to the general trauma ward. In total, he remained hospitalized for three days. At the time of discharge back to the prison infirmary, he was transitioned to oral amoxicillin/clavulanic acid, which was continued for two weeks post-op.

A CT scan three weeks following surgery showed an absence of cerebral edema, with resolution of the midline shift (Figure 5). The patient was scheduled for repeat head $\mathrm{CT}$ three months postoperatively, but refused imaging. He was seen in the neurosurgery clinic 16 weeks after surgery complaining of new onset left sided visual loss. On exam, the patient's pupils were fixed bilaterally. He was referred to neuro ophthalmology for further management of his visual complaints, but was ultimately lost to follow-up.

\section{Discussion}

Transorbital penetrating injuries (TOPI) are rare in the adult population, accounting for $0.096 \%$ of all head injuries [1-4]. In the setting of TOPI, there should be a high index of suspicion for damage to the intracrani-
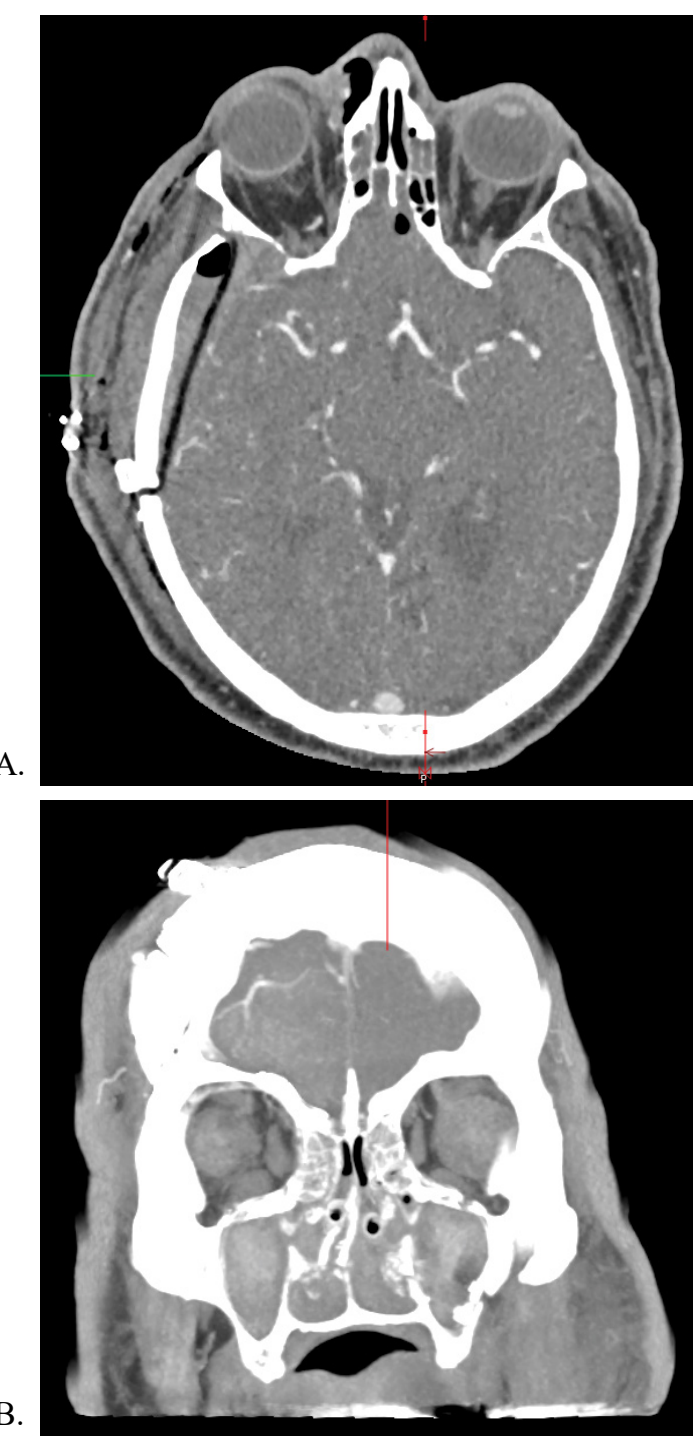

Figure 4: Immediate postoperative CTA following removal of foreign bodies.

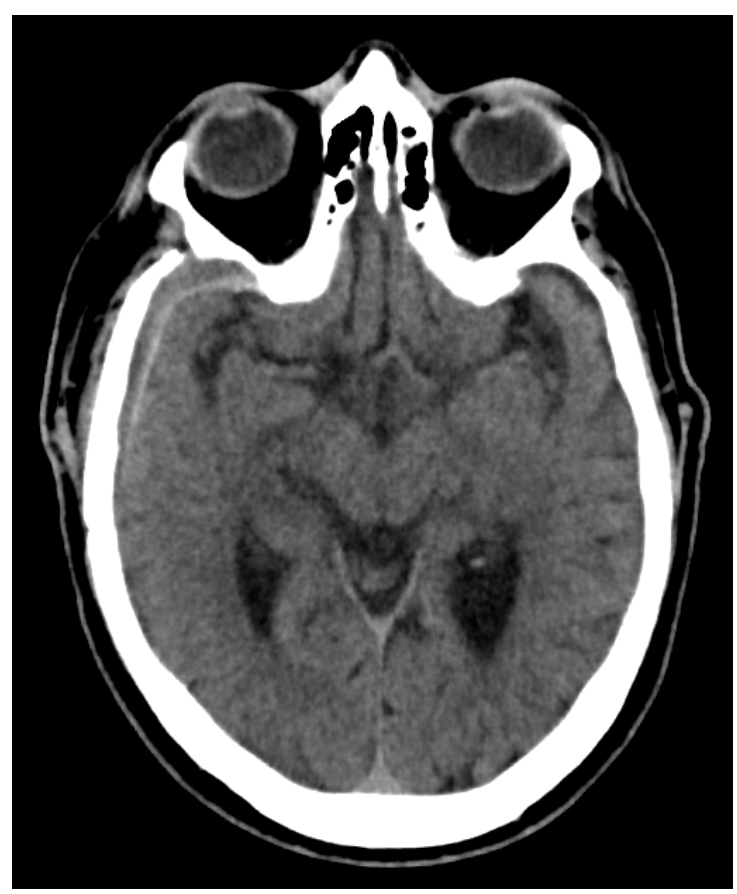

Figure 5: CT at three week postoperative mark. 
al vascular and neural structures. The globe, owing to a tough sclera and its relative mobility housed within fatty tissue, may remain intact despite brain parenchymal involvement $[1,5]$. The most common zone of entry is through a fracture of the thin walled superior orbital roof into the anterior cranial fossa $[2,3,6]$. This is thought to be related to reflexive extension of the neck at the time of injury, which results in a more caudal-to-rostral trajectory of the penetrating object [3]. Penetrating objects may enter the intracranial space without bony fracture through one of three posterior apertures: The superior orbital fissure, optic canal, and the inferior orbital fissure [1-8].

In a retrospective review of twenty one patients having suffered TOPI with the entry site being the medial zone of the eye, the most common sites of penetration into the cranium were as follows (in descending order): Superior orbital fissure, optic canal, sphenoid wing, and the orbital roof. The most common sites of central nervous system injury in medial zone TOPI, in descending order, were the cavernous sinus, temporal lobe, brainstem, frontal lobe, and the basal cisterns. The medial zone may be further compartmentalized into superior, central, and inferior subdivisions. The central subdivision corresponds to TOPI of the medial canthus, as occurred in the present case. For medial canthal TOPI, the most common sites of cranial penetration are the superior orbital fissure followed by the sphenoid wing. Furthermore, the most common sites of central nervous system injury following medial canthal TOPI are the cavernous sinus, followed by the temporal lobe, and then equal incidence of basal cistern and brainstem injury [1].

When patients present with medial zone TOPI, and complete loss of vision in the involved eye, injury to the optic nerve may be the culprit [1]. Some authors advocate prophylactic administration of anti-inflammatory glucocorticoids for optic nerve protection, though the clinical utility with respect to preservation of vision has not been elucidated $[5,7]$. Foreign body penetration of the superior orbital fissure characteristically results in ophthalmoplegia and reduced sensation in the distribution of the ophthalmic division of the trigeminal nerve, the so-called orbital apex syndrome [7]. Ocular paralysis may be explained by damage to the oculomotor, trochlear, and abducens nerves, all of which pass through the superior orbital fissure en route to their final sites of innervation. Alternatively, direct extraocular muscle injury may result in weakness, or a combination of both neural and muscular injury may be involved. Neural transection generally portends a poor prognosis for recovery, however, deficits related to retrobulbar hematoma formation or other mass lesions within the orbit should be considered surgical emergencies, as evacuation may result in resolution of ocular symptoms.

On presentation, a patient with TOPI should undergo an initial trauma survey. Following initial stabilization, cranial imaging, preferably non-contrasted head CT and CTA, should be performed to assess the degree of structural damage. These modalities aid in identification of object trajectory, the presence of bony fractures, the extent of damage to key vascular structures, and assist with operative planning [9]. In the present case, entry to the orbit was at the medial canthus, and entry into the intracranial compartment was through the superior orbital fissure [3,6-8]. No obvious cavernous sinus injury was seen on initial imaging evaluation. Even without CT evidence of injury, however, a high degree of suspicion must be maintained that the foreign body may be tamponading hemorrhage from a venous source or that artifact from metallic foreign bodies may obscure signs of injury on CT. Without adequate suspicion and planning, these occult injuries declare themselves during operative manipulation as uncontrolled hemorrhage [8]. As part of our surgical plan, we prepped the neck for possible cut down and temporary occlusion of the ipsilateral cervical carotid artery. Although CTA demonstrated no evidence of vascular injury, we did note damage to the adventitia of the cavernous carotid artery with direct visualization under the operative microscope.

Following initial removal of the foreign object, the patient must be monitored for further complications. Usually presenting around three to six weeks postoperatively, infection is the most complication of TOPI, and may manifest as meningitis, encephalitis, or cerebral abscess $[2,7,9]$. Some authors have advocated for the implementation of broad spectrum antibiotics in all cases of penetrating brain injury $[5,7]$. In the present case, the patient was started on ampicillin/sulbactam immediately following surgery. Additional delayed complications include cavernous sinus thrombosis, carotid-cavernous fistula, carotid aneurysm or pseudoaneurysm, and other vascular abnormalities. For these reasons, follow-up vascular imaging is recommended $[3,7,8]$.

One peculiarity in the current case was the patient's loss of contralateral vision. One possible explanation for this is the development of sympathetic ophthalmia, though no direct ocular injury was ever discovered in this case. He was referred for ophthalmological evaluation, but was lost to follow-up.

\section{Disclosure}

The authors have no disclosures to report.

\section{Authors Contribution}

All authors have contributed equally to the work.

\section{References}

1. RE Turbin, DN Maxwell, PD Langer, LP Frohman, B Hubbi L, et al. (2006) Patterns of transorbital intracranial injury: A review and comparison of occult and non-occult cases. Surv Ophthalmol 51: 449-460.

2. A Abdulbaki, F Al-Otabi, A Almalki, N Alohaly, S Baeesa 
(2012) Transorbital craniocerebral occult penetrating injury with cerebral abscess complication. Case Reports in Opthalmological Medicine.

3. YO Arat, A Arat, K Aydin (2015) Cerebrovascular complications of transorbital penetrating intracranial injuries. Ulus Travma Acil Cerrahi Derg.

4. A Singh, SK Bhasker, BK Singh (2013) Transorbital penetrating brain injury with a large foreign body. J Ophthalmic Vis Res.

5. Y Su, C Changchien (2016) Self-inflicted, trans-optic canal, intracranial penetrating injury with a ballpoint pen. J Surg Case Rep.
6. T Yamashita, T Mikami, T Baba, Y Minamida, TS ugino, et al. (2007) Transorbital intracranial penetrating injury from impaling on an earpick. Journal of Neuro-opthalmology.

7. M Schreckinger, D Orringer, BG Thompson, F La Marca, O Sagher (2011) Transorbital penetrating injury: Case series, review of the literature, and proposed management algorithm. J Neurosurg.

8. DR Lefebrve, RV Chandra (2012) Diagnosis and treatment of penetrating orbital cranial foreign bodies. Digit J Ophthalmol.

9. MA Rana, A Alharthy, WT Aletreby, B Huwait, A Kulshrestha (2014) Transorbital stab injury with retained knife: A narrow escape. Case Rep Crit Care. 